\title{
Antimicrobial resistance and antibiotic consumption in intensive care units, Switzerland, 2009 to 2018
}

Stefanie Barnsteiner ${ }^{1}$, Florent Baty ${ }^{2}$, Werner C Albrich ${ }^{1}$, Baharak Babouee Flury', ${ }^{1,3}$ Michael Gasser ${ }^{4}$, Catherine Plüss-Suard 4 , Matthias Schlegel ${ }^{1}$, Andreas Kronenberg ${ }^{4}$, Philipp Kohler ${ }^{1}$, on behalf of the Swiss Centre for Antibiotic Resistance (ANRESIS) ${ }^{4}$

1. Division of Infectious Diseases and Hospital Epidemiology, Cantonal Hospital St. Gallen, St. Gallen, Switzerland

2. Lung Center, Cantonal Hospital St. Gallen, St. Gallen, Switzerland

3. Medical Research Center, Cantonal Hospital St. Gallen, St. Gallen, Switzerland

4. Institute for Infectious Diseases, University of Bern, Bern, Switzerland

Correspondence: Philipp Kohler (philipp.kohler@kssg.ch)

Citation style for this article:

Barnsteiner Stefanie, Baty Florent, Albrich Werner C, Babouee Flury Baharak, Gasser Michael, Plüss-Suard Catherine, Schlegel Matthias, Kronenberg Andreas, Kohler Philipp, on behalf of the Swiss Centre for Antibiotic Resistance (ANRESIS). Antimicrobial resistance and antibiotic consumption in intensive care units, Switzerland, 2009 to 2018. Euro Surveill. 2021;26(46):pii=2001537. https://doi.org/10.2807/1560-7917.ES.2021.26.46.2001537

Background: Intensive care units (ICU) constitute a high-risk setting for antimicrobial resistance (AMR). Aim: We aimed to describe secular AMR trends including meticillin-resistant Staphylococcus aureus (MRSA), glycopeptide-resistant enterococci (GRE), extendedspectrum cephalosporin-resistant Escherichia coli (ESCR-EC) and Klebsiella pneumoniae (ESCR-KP), carbapenem-resistant Enterobacterales (CRE) and Pseudomonas aeruginosa (CRPA) from Swiss ICU. We assessed time trends of antibiotic consumption and identified factors associated with CRE and CRPA. Methods: We analysed patient isolate and antibiotic consumption data of Swiss ICU sent to the Swiss Centre for Antibiotic Resistance (2009-2018). Time trends were assessed using linear logistic regression; a mixed-effects logistic regression was used to identify factors associated with CRE and CRPA. Results: Among 52 ICU, MRSA decreased from $14 \%$ to $6 \%(p=0.005$; $n=6,465) ;$ GRE increased from $1 \%$ to $3 \%(p=0.011$; $\mathrm{n}=4,776)$. ESCR-EC and ESCR-KP increased from $7 \%$ to $15 \%(p<0.001, n=10,648)$ and $5 \%$ to $11 \%(p=0.002$; $\mathrm{n}=4,052)$, respectively. CRE, mostly Enterobacter spp., increased from $1 \%$ to $5 \%(p=0.008 ; n=17,987)$; CRPA remained stable at $27 \%(p=0.759 ; n=4,185)$. Antibiotic consumption in 58 ICU increased from 2009 to 2013 (82.5 to 97.4 defined daily doses (DDD)/100 bed-days) and declined until 2018 (78.3 DDD/100 beddays). Total institutional antibiotic consumption was associated with detection of CRE in multivariable analysis (odds ratio per DDD: 1.01 ; $95 \%$ confidence interval: 1.0-1.02; $p=0.004)$. Discussion: In Swiss ICU, antibiotic-resistant Enterobacterales have been steadily increasing over the last decade. The emergence of CRE, associated with institutional antibiotic consumption, is of particular concern and calls for reinforced surveillance and antibiotic stewardship in this setting.

\section{Introduction}

Intensive care units (ICU) are considered high-risk settings for antimicrobial resistance (AMR) [1]. This can be explained by an increased risk for nosocomial infections in ICU patients who have underlying comorbidities and frequently use of medical devices, but also by potentially increased exposure to resistant organisms where there is high patient turnover and by the large number of transmission opportunities [2]. Another particularity of the ICU setting is the extensive use of broad-spectrum antibiotics, which is considered to be one of the main drivers for the development and dissemination of $A M R$, in particular for resistant Gramnegative pathogens [3]. Indeed, several studies have reported an association between carbapenem consumption and the emergence of carbapenem-resistant Enterobacterales (CRE) and Pseudomonas aeruginosa (CRPA) $[4,5]$. The CRE and CRPA are pathogens of particular interest, as both have been classified by the World Health Organisation (WHO) as critical pathogens regarding the need for new antibiotic substances [6].

Surveillance is considered a cornerstone in the fight against AMR [7]. Specific surveillance data from AMR in Swiss ICU have not been published so far, although outbreaks caused by resistant pathogens have been reported [8]. Data from the Swiss Centre for Antibiotic Resistance (ANRESIS) for non-ICU settings have shown a steady rise of AMR among Gram-negative pathogens between 2007 and 2017, while meticillin-resistant Staphylococcus aureus (MRSA) are declining [9,10]. Antibiotic consumption in Swiss ICU has been shown to be somewhat lower than in other European countries; however, these data were collected between 2004 and 2008 [11].

In this study, we describe secular trends of AMR and antibiotic consumption from Swiss ICU using surveillance data from ANRESIS. For CRE and CRPA, we sought 
to identify patient-level and institutional factors associated with resistance, including institutional antibiotic consumption. We also assessed the representativeness of ANRESIS for the ICU setting in Switzerland.

\section{Methods}

\section{Data source}

This laboratory-based surveillance study was conducted using antimicrobial resistance and antibiotic consumption data from ANRESIS (www.anresis.ch). ANRESIS, led by the Institute for Infectious Diseases at the University of Bern, is the national centre for surveillance of antibiotic resistance and antibiotic consumption and is supported by the Swiss Federal Office of Public Health. Antimicrobial susceptibility testing is performed at local laboratories according to guidelines from the Clinical and Laboratory Standards Institute (CLSI) or the European Committee on Antimicrobial Susceptibility Testing (EUCAST) [12,13]. All laboratories are accredited by Swiss authorities and participate in at least one external quality programme of either the National External Quality Assessment Service (https://ukneqas.org.uk) or the Swiss quality control programme of the Institute for Medical Microbiology, University of Zurich (www.imm.uzh.ch/de/services/ qc.html).

\section{Representativeness of ANRESIS}

ANRESIS continuously collects routine antibiotic resistance data (along with basic patient information including age category, sex and sample location) from 33 laboratories distributed homogeneously all over Switzerland and representing $89 \%$ of all hospital samples in 2020. Antibiotic consumption data are collected once yearly from around 70 hospital pharmacies across Switzerland. For the analysis of AMR in ICU, we assessed the representativeness of ANRESIS for ICU registered by the Swiss Society for Intensive Care (www.sgi-ssmi.ch/de/zertifizierte-is.html). Because several small-size ICU have been closed or have been incorporated into larger institutions over the last decade, representativeness was only assessed for the year 2018. This was done by calculating the percentage of ICU beds represented in ANRESIS among all registered ICU beds in Switzerland, stratified by administrative subdivisions (i.e. cantons) speaking mostly French or Italian (cantons of Fribourg, Geneva, Jura, Neuchâtel, Ticino, Valais and Vaud; henceforth referred to as south-west) or German (all other cantons; henceforth referred to as east). In order to detect differences in microbiological sampling frequencies between geographical regions, we calculated the sampling density, defined as the number of microbiological samples per represented ICU bed.

Inclusion criteria for antimicrobial resistance and antibiotic consumption data

For the analysis of AMR, we extracted microbiological isolates with the label 'intensive care' sent to ANRESIS between January 2009 and December 2018 from patients 15 years and older. Although data were anonymous, every patient possesses a unique identity number within every institution, which was used to exclude repeat isolates (i.e. same pathogen and resistance profile) from the same patient in a specific calendar year. If patients contributed both resistant and susceptible isolates (per species) in the same year, only the resistant isolate was included. Therefore, each patient contributed only one sample per species, institution and year. Bacterial isolates from non-specified swabs (presumably rectal swabs taken at time of admission) were excluded in order to minimise the impact of patients colonised with resistant pathogens before ICU admission.

For the analysis of antibiotic consumption, we extracted consumption data for the same time period (2009 to 2018) from those ICU where these data were available. of note, antibiotic consumption data were only available on the institutional level (per year) and not on the patient level.

For the analysis of risk factors for CRE and CRPA, we included only data from years where institutions provided AMR data along with corresponding antibiotic consumption data.

\section{Definitions for antimicrobial resistance}

Resistance to a particular substance was defined as either an intermediate or a resistant phenotype in susceptibility testing according to the individual laboratories. Group resistance (e.g. glycopeptide, carbapenem, aminoglycoside or quinolone resistance) was defined as resistance or intermediate susceptibility to at least one antibiotic of the respective group; extended-spectrum cephalosporin-resistance was defined as resistance or intermediate susceptibility to at least one third or fourth generation cephalosporin.

Among Gram-positive bacteria, we analysed MRSA and glycopeptide-resistant Enterococcus faecalis/faecium (GRE). Non-multidrug-resistant MRSA was used as a surrogate for community-associated MRSA (caMRSA), defined as being susceptible to at least three of the following agents: ciprofloxacin, clindamycin, tetracycline and trimethoprim-sulfamethoxazole [10]. The following Gram-negative pathogens were analysed: extendedspectrum cephalosporin-resistant Escherichia coli (ESCR-EC) and Klebsiella pneumoniae (ESCR-KP), CRE (of note, species with intrinsically low activity against imipenem such as Proteus spp., Providencia spp. or Morganella spp. were excluded), CRPA (of note, only resistance to imipenem or meropenem was considered), carbapenem-resistant Acinetobacter baumannii complex (CRAB) and trimethoprim-sulfamethoxazoleresistant Stenotrophomonas maltophilia (TSRSM).

\section{Data analysis and statistics}

We expressed AMR as a percentage of all isolates and consumption of systemic antibiotics as defined daily doses (DDD) per 100 bed-days (BD), compliant with the 
World Health Organization (WHO) definition from 2019 [14]. Categorical variables were reported as frequencies and proportions, continuous variables as median with interquartile range (IQR). For dichotomous variables, we used chi-squared or Fisher's exact test, as appropriate. For continuous variables, we used the Wilcoxon rank-sum test. For each type of resistance, the proportion of pathogens with the resistance trait (as percentage of the total number of isolates reported and tested for the respective key antibiotic) was analysed over time. Linear regression analysis was used to estimate temporal trends, using resistance or antibiotic consumption as dependent and year of surveillance as independent variable.

For the analysis of risk factors for CRE and CRPA, we performed a mixed model with ICU as random effect, considering the hierarchical data structure. Patientlevel variables included age group $(\langle 60,60-75$ and $\rangle 75$ years), sex and site of sample collection (blood, respiratory, urogenital or other). Institutional variables included geographical region (south-west vs east), hospital type (university vs non-university hospitals) and total annual antibiotic consumption. All co-variables were entered into multivariable analysis. Two-sided $p$ values $\leq 0.05$ were considered statistically significant. All statistical analyses were performed using $R$ statistical software version 3.5.2 (R Foundation, Vienna, Austria).

\section{Sensitivity analyses}

Sensitivity analyses were performed for the assessment of temporal trends for AMR and antibiotic consumption, including only those ICU that provided data for at least 8 years between 2009 and 2018, in order to eliminate potential selection bias.

\section{Ethical statement}

Because ANRESIS contains routinely collected anonymised surveillance data, ethical consent was not required according to the Swiss law for research on human beings.

\section{Results}

\section{Representativeness of included intensive care}

units

Over the 10-year period, we included 52 and 58 ICU for the analysis of AMR and antibiotic consumption, respectively (for an overview see Supplementary Figure S1). By 2018, 11 of 52 ICU did no longer exist, resulting in $41 \mathrm{ICU}$ represented in our database for the analysis of AMR. Of these 41 ICU (with a median bed number of nine), 17 were located in south-western, and 24 in eastern Switzerland; the median bed number was 39 (range: 34-64) for the five university and eight (range: 6-42) for the 36 non-university ICU (listed in Supplementary Table S1).

The 41 ICU covered $55 \%$ of the 74 registered ICU in Switzerland for the year 2018 , or $70 \%(611 / 871)$ of all
Swiss ICU beds. Coverage was higher for south-western (203/237; 86\%) than for eastern Switzerland (408/634; $64 \%)(p<0.001)$. For the year 2018 , sampling density was 10.2 samples per represented ICU bed; sampling density was higher for ICU from the south-west $(12.2$ per represented bed) compared with those from the east (9.3 per represented bed) $(p=0.002)$ (Figure 1).

\section{Antimicrobial resistance}

After removal of duplicates, we identified 34,887 tested pathogens. A majority of isolates $(n=19,571$; $56 \%$ ) originated from eastern and 15,316 (44\%) from south-western Switzerland. Resistance data by geographical region and year (2009 and 2018) are shown in Table 1; temporal trends of bacterial resistance are shown in Figure 2 (and in Supplementary Figures $\mathrm{S}_{2} / \mathrm{S}_{3}$ for eastern and south-western Switzerland separately).

Among the $S$. aureus isolates ( $n=6,465$; median: 654 / year; range: 530-732), meticillin resistance decreased from $14 \%$ to $6 \%(p=0.005)$ from 2009 to 2018 . This effect was driven by a decline in hospital-associated MRSA (haMRSA) in south-western Switzerland from $18 \%$ to $2 \%$ (p $<0.001$ ); the proportion of caMRSA was stable around $3 \%(p=0.196)$. Among the 4,776 enterococcal isolates (median: 478/year; range: $282-627$ ), GRE increased from $1 \%$ to $3 \%(p=0.011)$, which was exclusively due to an increase in eastern Switzerland.

We identified 10,648 E. coli (median: 1,019/year: range: 817-1,317) and 4,052 K. pneumoniae (median: 394/

\section{FIGURE 1}

Intensive care units represented in the analysis of antimicrobial resistance, Switzerland, 2009-2018 $(n=41)$

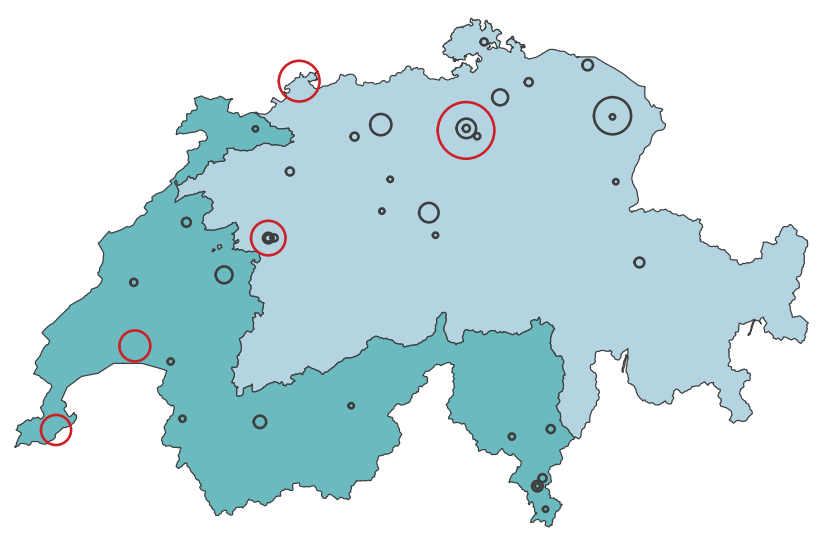

$\square$ Eastern Switzerland (coverage 64\%, 9.3 samples per bed)
Western Switzerland (coverage 86\%, 12.2 samples per bed)
University hospitals
Non-university hospitals

The map is limited to institutions registered in 2018. Circle sizes represent number of samples. Coverage (i.e. number of beds of all registered beds) and sampling density (i.e. number of microbiological samples per represented beds) are given by geographical region. 
year; range: $328-471$ ) isolates. From 2008 to 2019, ESCR for $E$. coli increased from $7 \%$ to $15 \%(p<0.001)$ and for K. pneumoniae from $5 \%$ to $11 \%(\mathrm{p}=0.002)$. Proportions and trends for ESCR were similar between the two geographical regions. The CRE showed an increase from $1 \%$ to $5 \%(p=0.008$; total isolates: $n=17,987)$ and were more common in eastern Switzerland ( $5 \%$ vs $3 \%$, p $<0.001)$. In 2009, 16 of 17 CRE were Enterobacter spp., whereas in 2018, $77 \%$ (82/107) were Enterobacter spp., $11 \%(12 / 107)$ were $K$. pneumoniae, and $8 \%(9 / 107)$ were E. coli. Among 4,185 P. aeruginosa isolates (median: 414/year; range: $381-465$ ), $27 \%$ were CRPA, with no change over time $(p=0.759)$. Isolates of the A. baumannii complex were rarely detected $(n=316) ; 37 \%$ of these isolates were CRAB, without temporal change $(p=0.495)$. Also for the 1,158 S. maltophilia isolates, of which $4 \%$ were TSRSM, no trend could be observed $(p=0.091)$.

\section{Antibiotic consumption}

Among 35 ICU from south-western and 23 from eastern Switzerland, penicillins, cephalosporins and carbapenems were the three antibiotic groups used the most. For 2018 , they accounted for $68 \%$ (52.9 DDD/100 BD) of the total (78.3 DDD/100 BD) antibiotic consumption (for a detailed list see Supplementary Table S2). Temporal trends of these antibiotic groups, for quinolones and for the total antibiotic consumption are shown in Figure 3 (and in Supplementary Figures $\mathrm{S}_{4} / \mathrm{S}_{5}$ stratified by geographical region). We observed an increase in total antibiotic consumption from 2009 (82.5 DDD/100 BD) to 2013 (97.4 DDD/100 BD), followed by a decrease until 2018 (78.3 DDD/100 BD). Segmented linear regression (performed post-hoc) confirmed these opposing trends $(p=0.044)$. Similar patterns were observed for the 'watch and reserve' group of antibiotics (WHO AWaRe classification) [15], as well as for carbapenems and quinolones. Consumption of piperacillin/tazobactam increased from 8.0 to 11.0 DDD per $100 \mathrm{BD}(p=0.003)$ from 2009 to 2018; ceftriaxone consumption increased from 6.3 to 7.9 DDD per 100 BD ( $p<0.001)$. Notable geographical differences were a lower carbapenem (11.4 vs 15.6 DDD/100 BD; $p=0.002$ ) and quinolone consumption (3.0 vs $4.0 \mathrm{DDD} / 100 \mathrm{BD} ; \mathrm{p}=0.014$ ) in eastern compared with south-western Switzerland (Supplementary Table S3).

\section{Risk factors for carbapenem resistance}

For CRE, institutional antibiotic consumption was significantly (but not very strongly) associated with carbapenem resistance in the mixed effects model (odds ratio (OR) per DDD: 1.01; 95\% confidence interval $(\mathrm{Cl})$ : $1.0-1.02 ; p=0.004)$, as was the year of detection ( $O R$ per year: $1.1 ; 95 \% \mathrm{Cl}: 1.1-1.2 ; \mathrm{p}<0.001)$. For CRPA, the association with antibiotic consumption was not statistically significant (OR: 1.00; 95\% Cl: 0.99-1.01; $\mathrm{p}=0.083$ ). Interestingly, male sex was positively (OR: 1.3; $95 \% \mathrm{Cl}: 1.1-1.6 ; p=0.002)$ and year of detection although with very small effect - negatively (OR: 0.99; 95\% Cl: 0.98-0.99; $\mathrm{p}<0.001)$ associated with CRPA. Older age and detection in urine were negatively associated with both CRE and CRPA. Neither geographical region nor university hospital status was predictive for CRE or CRPA (Table 2).

Sensitivity analyses for temporal trends Considering only those ICU that provided data for at least 8 years between 2009 and 2018, we observed the

TABLE 1

Number of resistant and total number of isolates from intensive care units, by pathogen, eastern vs south-western Switzerland, $2009(\mathrm{n}=3,187)$ and $2018(\mathrm{n}=4,030)$

\begin{tabular}{|c|c|c|c|c|c|c|}
\hline & \multicolumn{3}{|c|}{2009} & \multicolumn{3}{|c|}{2018} \\
\hline & East & South-west & \multirow{2}{*}{$p$ value } & East & South-west & \multirow{2}{*}{$p$ value } \\
\hline & $\mathrm{n} /$ all & $\mathrm{n} /$ all & & $\mathrm{n} /$ all & n/all & \\
\hline GRE & $1 / 132$ & $1 / 168$ & $>0.999^{*}$ & $14 / 333$ & $5 / 293$ & 0.113 \\
\hline ESCR-EC & $31 / 411$ & $35 / 528$ & 0.678 & $114 / 730$ & $65 / 500$ & 0.232 \\
\hline ESCR-KP & $11 / 139$ & $7 / 157$ & $>0.999$ & $34 / 280$ & $18 / 171$ & 0.439 \\
\hline CRE & $12 / 833$ & $5 / 729$ & 0.234 & $81 / 1,324$ & $26 / 851$ & 0.002 \\
\hline Enterobacter spp. & $11 / 12$ & $5 / 5$ & $\mathrm{Nd}$ & $64 / 81$ & $18 / 26$ & $\mathrm{Nd}$ \\
\hline Klebsiella pneumoniae & $0 / 12$ & $0 / 5$ & $\mathrm{Nd}$ & $9 / 81$ & $3 / 26$ & $\mathrm{Nd}$ \\
\hline Escherichia coli & $0 / 12$ & $0 / 5$ & $\mathrm{Nd}$ & $5 / 81$ & $4 / 26$ & $\mathrm{Nd}$ \\
\hline Other & $1 / 12$ & $0 / 5$ & $\mathrm{Nd}$ & $3 / 81$ & $1 / 26$ & $\mathrm{Nd}$ \\
\hline CRPA & $53 / 253$ & $65 / 209$ & 0.017 & $62 / 254$ & $39 / 171$ & 0.792 \\
\hline TSRSM & $4 / 65$ & $2 / 68$ & $0.434^{*}$ & $2 / 71$ & $1 / 57$ & >0.999* \\
\hline
\end{tabular}

CRAB: carbapenem-resistant Acinetobacter baumannii complex; CRE: carbapenem-resistant Enterobacterales; CRPA: carbapenem-resistant Pseudomonas aeruginosa; GRE: glycopeptid-resistant Enterococcus faecalis/faecium; ESCR-EC: extended-spectrum cephalosporin-resistant Escherichia coli; ESCR-KP: extended-spectrum cephalosporin-resistant Klebsiella pneumoniae; MRSA: meticillin-resistant Staphylococcus aureus; $\mathrm{Nd}$ : not done; TSRSM: trimethoprim-sulfamethoxazole-resistant Stenotrophomonas maltophilia.

Please note that $E$. coli and $K$. pneumoniae isolates were used for both the analysis on ESCR and carbapenem resistance. p values (chisquared or *Fisher's exact test ) $\leq 0.05$ were considered statistically significant (in bold). 
FIGURE 2

Trends in resistant pathogens in intensive care, Switzerland, 2009-2018 $(\mathrm{n}=34,887)$

A. MRSA $(n=6,465)$

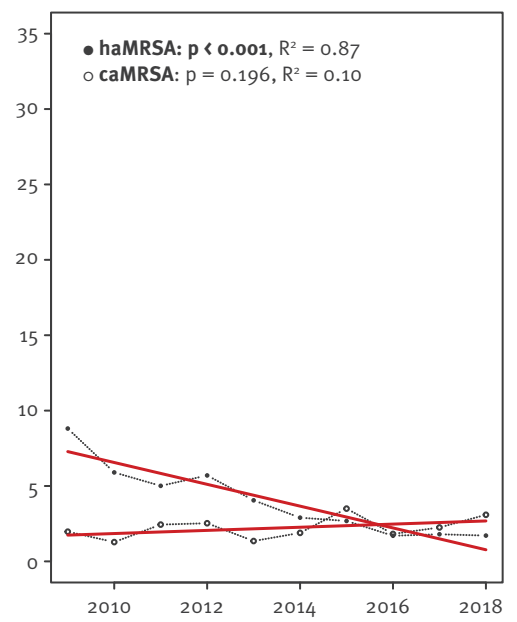

D. ESCR-KP $(n=4,052)$

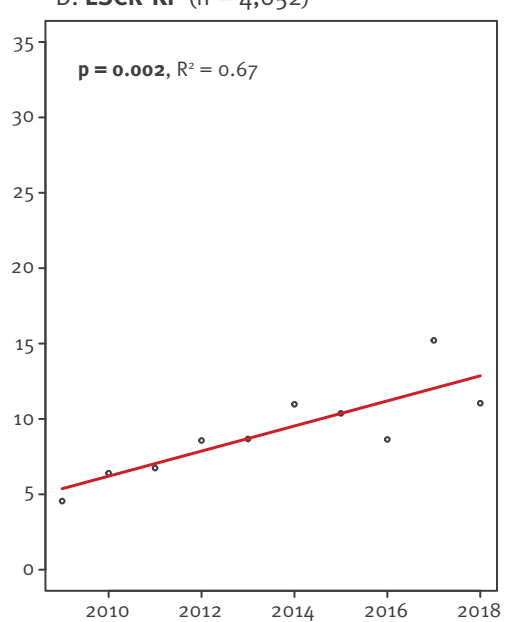

G. CRAB $(n=316)$

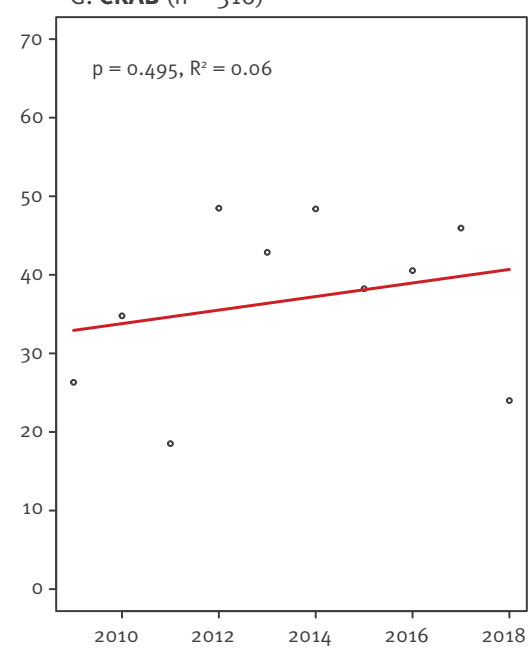

B. GRE $(n=4,776)$

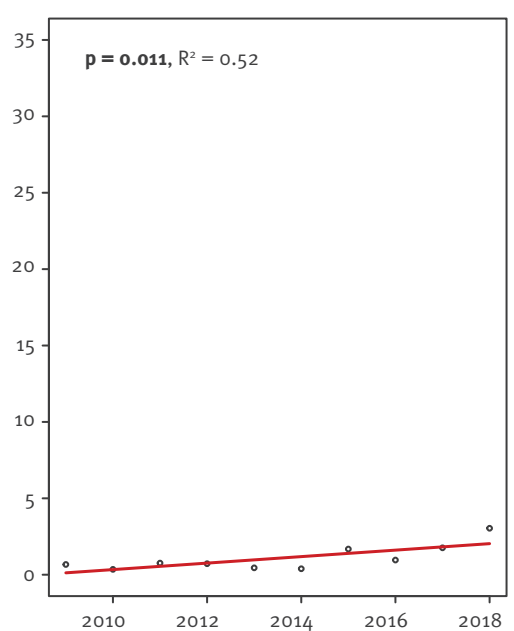

E. CRE $(n=17,987)$

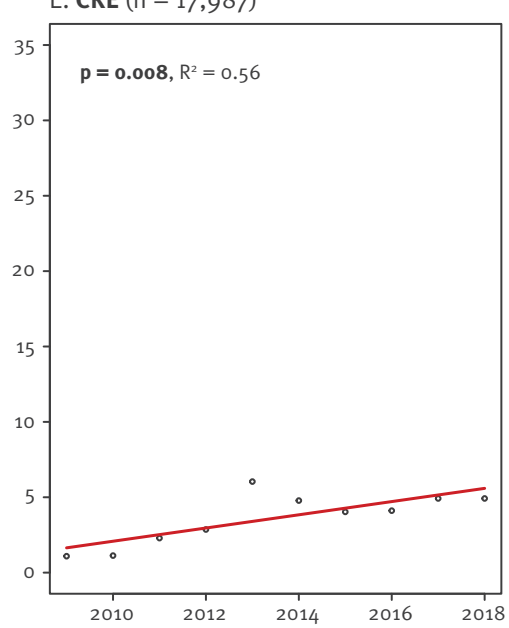

H. TSRSM $(n=1,158)$

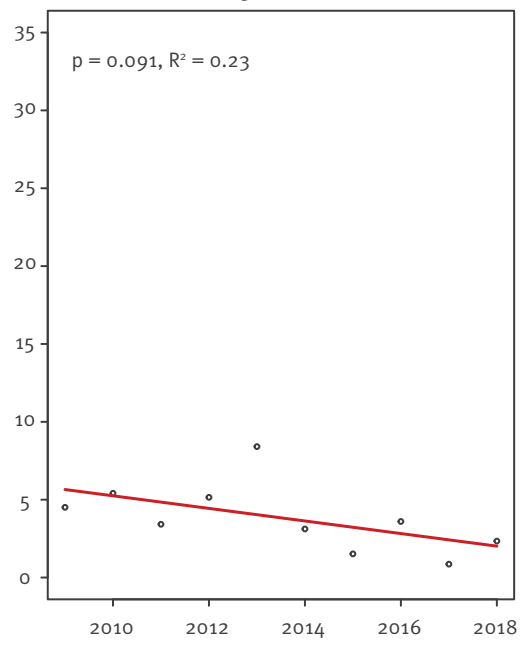

C. ESCR-EC $(n=10,648)$

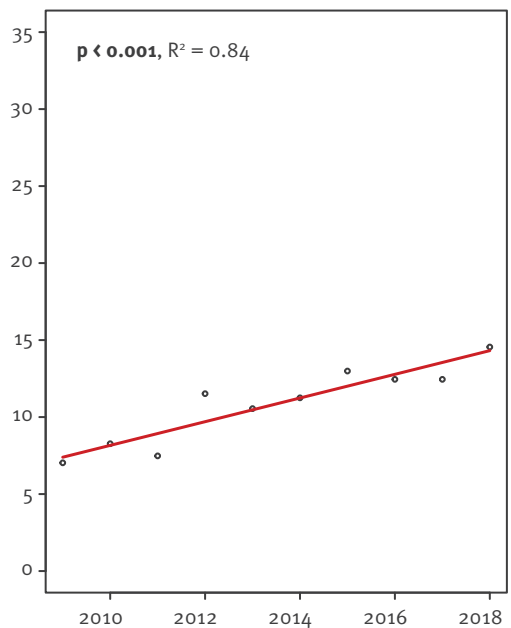

F. CRPA $(n=4,185)$

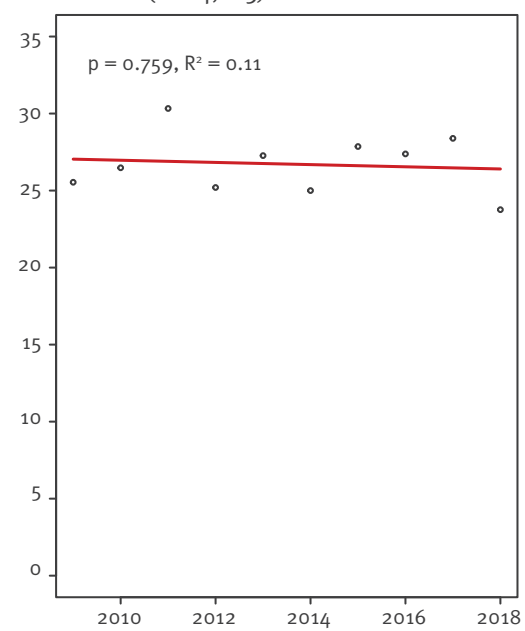

Year

CaMRSA: community-acquired MRSA; CRAB: carbapenem-resistant Acinetobacter baumannii complex; CRE: carbapenem-resistant

Enterobacterales; CRPA: carbapenem-resistant Pseudomonas aeruginosa; GRE: glycopeptid-resistant Enterococcus faecalis/faecium; ESCR-EC: extended-spectrum cephalosporin-resistant Escherichia coli; ESCR-KP: extended-spectrum cephalosporin-resistant Klebsiella pneumoniae; haMRSA: hospital-associated MRSA; MRSA: meticillin-resistant Staphylococcus aureus; TSRSM: trimethoprim-sulfamethoxazole-resistant Stenotrophomonas maltophilia.

$\mathrm{Y}$ axes show \% of resistant (note the different scale for the y axis in panel G). Please note that E. coli and $K$. pneumoniae isolates were used for both the analysis on ESCR and carbapenem resistance. The number of all isolates (sensitive and resistant) is indicated in brackets (n). $P$ values $\leq 0.05$ were considered statistically significant (in bold). 
same temporal trends regarding AMR (in total 28 ICU; 15 from the south-west, 13 from the east of Switzerland) and antibiotic consumption (in total 29 ICU; 14 from the south-west, 15 from the east of Switzerland) (data not shown).

\section{Discussion}

In this laboratory-based analysis of national surveillance data from Swiss ICU, we have shown that the occurrence of MRSA has been decreasing steadily, whereas resistant Gram-negative bacteria including ESCR-EC, ESCR-KP and - of particular concern - CRE have become more frequent between 2009 and 2018. The only modifiable factor associated with CRE was institutional antibiotic consumption. On a national level, antibiotic consumption peaked in 2013 and has since been declining. The strengths of this study were its large sample size, the representativeness of the database for the Swiss ICU setting and the robustness of temporal trends in sensitivity analyses.

\section{FIGURE 3}

Trends in overall antibiotic consumption and consumption of preselected antibiotic substances in intensive care, in defined daily doses per 100 bed-days, Switzerland, 2009-2018

A. Total antibiotic consumption

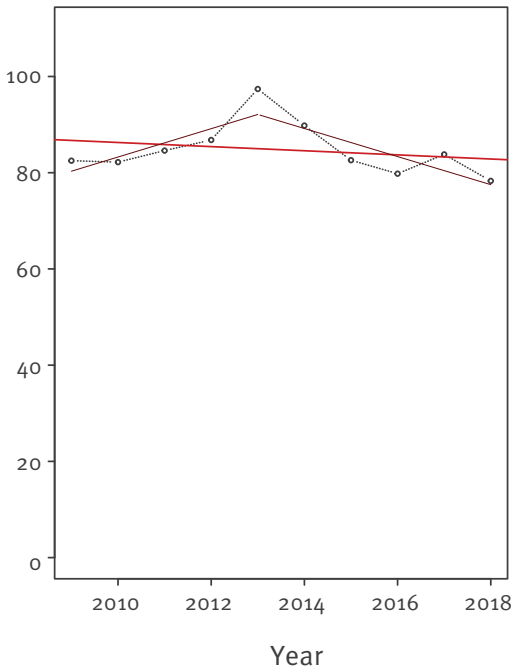

$p=0.512, R^{2}=0.006$

Change in slope: $p=0.044$

\section{Cephalosporins}

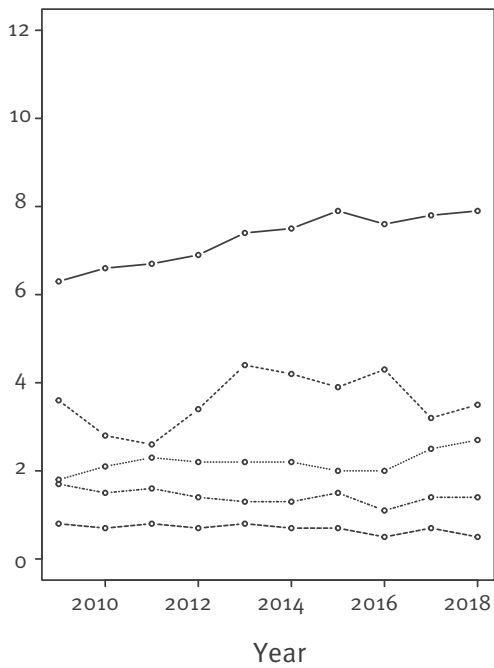

- Ceftriaxone -....... Cefazolin

-..-... Cefuroxime --.-- Ceftazidime

Cefepime
B. Carbapenems

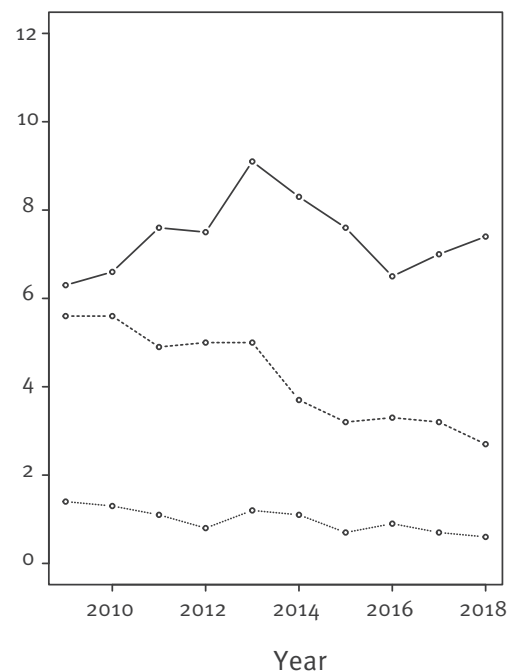

- Meropenem $\quad$ Ertapenem -.....-.- Imipenem

\section{E. Penicillins}

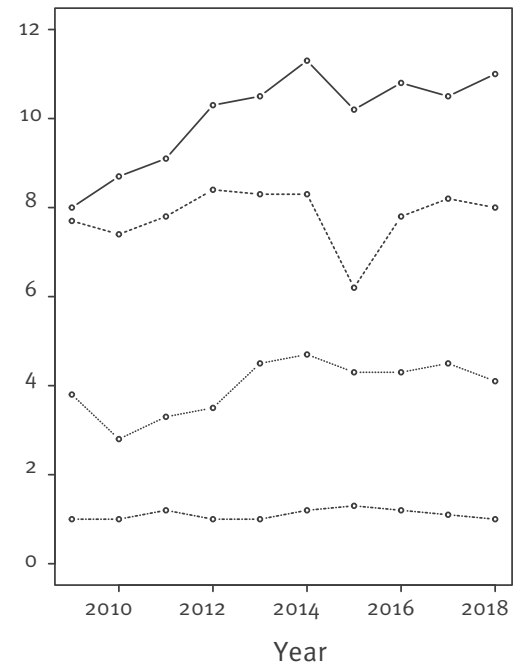

- Piperacillin/BLI ......... Flucloxacillin

....... Amoxicillin/BLI_..... Amoxicillin

\section{Quinolones}

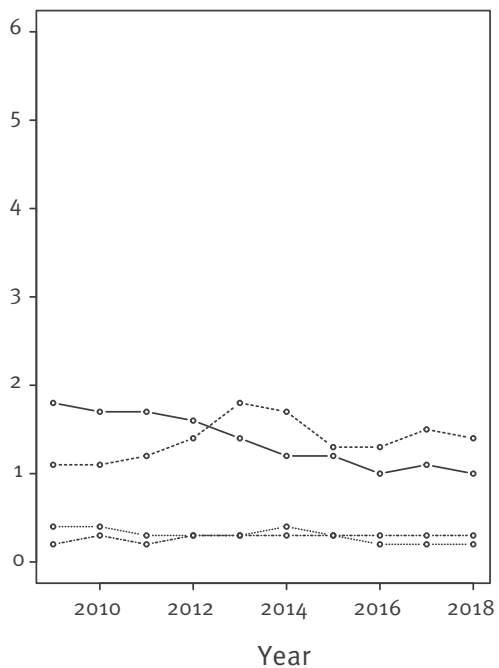

- Ciprofloxacin - Norfloxacin -...-... Levofloxacin Moxifloxacin 
TABLE 2

Factors associated with carbapenem-resistant Enterobacterales spp. (n = 14,479) and carbapenem-resistant Pseudomonas aeruginosa $(\mathrm{n}=3,512)$ in intensive care, Switzerland, 2009-2018

\begin{tabular}{|c|c|c|c|c|c|c|c|c|}
\hline \multirow{2}{*}{ Covariables } & \multicolumn{2}{|c|}{ Sensitive } & \multicolumn{2}{|c|}{ Resistant } & \multicolumn{2}{|c|}{ Univariable } & \multicolumn{2}{|c|}{ Multivariable } \\
\hline & $\mathrm{n}$ & $\%$ & $\mathrm{n}$ & $\%$ & OR $(95 \% \mathrm{Cl})$ & $p$ value & OR $(95 \% \mathrm{Cl})$ & $p$ value \\
\hline Enterobacterales spp. & \multicolumn{2}{|c|}{$n=13,884$} & \multicolumn{2}{|c|}{$n=595$} & & & & \\
\hline \multicolumn{9}{|l|}{ Year } \\
\hline OR per year & $\mathrm{Nd}$ & $\mathrm{Nd}$ & $\mathrm{Nd}$ & $\mathrm{Nd}$ & $1.13(1.13-1.14)$ & $<0.001$ & $1.12(1.10-1.12)$ & $<0.001$ \\
\hline \multicolumn{9}{|l|}{ Age } \\
\hline $15-<60$ & 4,046 & 29 & 207 & 35 & Ref & Ref & Ref & Ref \\
\hline $60-75$ & 7,440 & 54 & 321 & 54 & $0.9(0.8-1.1)$ & 0.539 & $0.9(0.8-1.1)$ & 0.441 \\
\hline$>75$ & 2,398 & 17 & 67 & 11 & $0.6(0.5-0.8)$ & 0.002 & $0.7(0.5-0.9)$ & 0.006 \\
\hline \multicolumn{9}{|l|}{ Sex } \\
\hline Female & 5,284 & 38 & 189 & 32 & Ref & Ref & Ref & Ref \\
\hline Male & 8,600 & 62 & 406 & 68 & $1.3(1.1-1.6)$ & 0.001 & $1.0(0.8-1.2)$ & 0.838 \\
\hline \multicolumn{9}{|l|}{ Sample localisation } \\
\hline Blood culture & 1,294 & 9 & 47 & 8 & Ref & Ref & Ref & Ref \\
\hline Respiratory sample & 6,854 & 49 & 349 & 59 & $1.2(0.9-1.7)$ & 0.194 & $1.3(0.9-1.7)$ & 0.146 \\
\hline Urine & 3,360 & 24 & 60 & 10 & $0.4(0.3-0.6)$ & $<0.001$ & $0.4(0.3-0.6)$ & $<0.001$ \\
\hline Other & 2,376 & 17 & 139 & 23 & $1.8(1.3-2.5)$ & 0.001 & $1.8(1.3-2.5)$ & 0.001 \\
\hline \multicolumn{9}{|l|}{ Region } \\
\hline Eastern Switzerland & 7,577 & 55 & 407 & 68 & Ref & Ref & Ref & Ref \\
\hline South-western Switzerland & 6,307 & 45 & 188 & 32 & $1.1(0.6-2.3)$ & 0.717 & $1.2(0.6-2.4)$ & 0.570 \\
\hline \multicolumn{9}{|l|}{ University status } \\
\hline Non-university hospital & 6,848 & 49 & 200 & 34 & Ref & Ref & Ref & Ref \\
\hline University hospital & 7,036 & 51 & 395 & 66 & $1.1(0.5-2.5)$ & 0.834 & $0.9(0.6-2.1)$ & 0.842 \\
\hline \multicolumn{9}{|l|}{ Total antibiotic consumption } \\
\hline DDD/10oBD, median (IQR) & 98 & $87 ; 116$ & 115 & $91 ; 142$ & $1.02(1.01-1.02)$ & $<0.001$ & $1.01(1.00-1.02)$ & 0.004 \\
\hline Pseudomonas aeruginosa & \multicolumn{2}{|c|}{$n=2,521$} & \multicolumn{2}{|c|}{$n=991$} & & & & \\
\hline \multicolumn{9}{|l|}{ Year } \\
\hline OR per year & $\mathrm{Nd}$ & $\mathrm{Nd}$ & $\mathrm{Nd}$ & $\mathrm{Nd}$ & $0.98(0.92-1.04)$ & 0.502 & $0.99(0.98-0.99)$ & $<0.001$ \\
\hline \multicolumn{9}{|l|}{ Age } \\
\hline $15-<60$ & 641 & 25 & 400 & 40 & Ref & Ref & Ref & Ref \\
\hline $60-75$ & 1,447 & 57 & 513 & 52 & $0.6(0.5-0.7)$ & $<0.001$ & $0.6(0.5-0.7)$ & $<0.001$ \\
\hline$>75$ & 433 & 17 & 78 & 8 & $0.3(0.2-0.4)$ & $<0.001$ & $0.4(0.3-0.5)$ & $<0.001$ \\
\hline \multicolumn{9}{|l|}{ Sex } \\
\hline Female & 876 & 35 & 286 & 29 & Ref & Ref & Ref & Ref \\
\hline Male & 1,645 & 65 & 705 & 71 & $1.3(1.1-1.6)$ & $<0.001$ & $1.3(1.1-1.6)$ & 0.002 \\
\hline \multicolumn{9}{|l|}{ Sample localisation } \\
\hline Blood & 128 & 5 & 54 & 5 & Ref & Ref & Ref & Ref \\
\hline Respiratory & 1,546 & 61 & 663 & 67 & $1.1(0.8-1.5)$ & 0.741 & $1.0(0.7-1.5)$ & 0.812 \\
\hline Urine & 471 & 19 & 87 & 9 & $0.5(0.3-0.7)$ & $<0.001$ & $0.5(0.3-0.8)$ & $<0.001$ \\
\hline Other & 376 & 15 & 187 & 19 & $1.2(0.8-1.7)$ & 0.384 & $1.2(0.8-1.7)$ & 0.377 \\
\hline \multicolumn{9}{|l|}{ Region } \\
\hline Eastern Switzerland & 1,472 & 58 & 512 & 52 & Ref & Ref & Ref & Ref \\
\hline South-western Switzerland & 1,049 & 42 & 479 & 48 & $1.4(0.9-2.2)$ & 0.086 & $1.4(0.9-2.0)$ & 0.057 \\
\hline University status & & & & & & & & \\
\hline Non-university hospital & 1,193 & 47 & 355 & 36 & Ref & Ref & Ref & Ref \\
\hline University hospital & 1,328 & 53 & 636 & 64 & $1.6(1.0-2.5)$ & 0.060 & $1.3(0.8-1.9)$ & 0.262 \\
\hline Total antibiotic consumption & & & & & & & & \\
\hline DDD/10oBD, median (IQR) & 98 & $86 ; 115$ & 105 & $89 ; 125$ & $1.00(0.99-1.01)$ & 0.205 & $1.00(0.99-1.01)$ & 0.083 \\
\hline
\end{tabular}

CRAB: carbapenem-resistant Acinetobacter baumannii complex; CRE: carbapenem-resistant Enterobacterales; CRPA: carbapenem-resistant Pseudomonas aeruginosa; GRE: glycopeptid-resistant Enterococcus faecalis/faecium; ESCR-EC: extended-spectrum cephalosporin-resistant Escherichia coli; ESCR-KP: extended-spectrum cephalosporin-resistant Klebsiella pneumoniae; MRSA: meticillin-resistant Staphylococcus aureus; Nd: not done; TSRSM: trimethoprim-sulfamethoxazole-resistant Stenotrophomonas maltophilia.

Please note that E. coli and K. pneumoniae isolates were used for both the analysis on ESCR and carbapenem resistance. $p$ values (chisquared or *Fisher's exact test ) $\leq 0.05$ were considered statistically significant (in bold). 
Our main finding was the emergence of resistance among Enterobacterales in Swiss ICU over the last decade. These data are in line with trends described from other European countries, although the different observation periods overlap only partly with our analysis. In German ICU, ESCR-EC increased from 1.3\% to $16.3 \%$ and imipenem-resistant $K$. pneumoniae from $0.4 \%$ to $1.6 \%$ between 2001 and 2015 [16]. In France, ESBL-producing Enterobacterales increased by $73 \%$ between 2009 and 2013, with higher rates in ICU [17]. In Italy, bloodstream infections caused by CPE, mainly originating in ICU, increased steadily between 2014 and 2017 [18]. Resistant Gram-negative pathogens are also increasing in Swiss non-ICU settings, with up to $22 \%$ of $E$. coli being ESCR-EC in long-term care institutions $[19,20]$.

The emergence of CRE, associated with increased morbidity and mortality, is particularly worrying [21]. Enterobacter spp. were the predominant species among our CRE. Carbapenem-resistance in Enterobacter spp. can be conferred either by de-repressed AmpC in combination with porin loss/modification or by carbapenemase production $[22,23]$. De-repression of $A m p C$ in Enterobacter spp. is usually observed after beta-lactam exposure, which is in accordance with our finding that institutional antibiotic consumption was associated - although not very strongly - with detection of CRE. However, we cannot exclude the possibility that the rise in carbapenem-resistant Enterobacter spp. may be due to dissemination of high-risk clones such as ST171 and $\mathrm{ST}_{78}$, which can also carry carbapenemase-encoding plasmids [23]. Another argument suggesting the emergence of CPE in Swiss ICU is the fact that antibiotic consumption, including carbapenem and quinolone consumption, is declining and that $K$. pneumoniae and $E$. coli are increasing among CRE. Increased importation of CPE from high-risk countries or even acquisition in local hospitals could account for their spread, as CPE are considered to spread primarily through horizontal transmission in the healthcare setting $[8,24,25]$. For instance, the hospital water environment (including drains and sinks) has increasingly been recognised as a reservoir for outbreaks of carbapenem-resistant organisms in the ICU setting [26]. Also, national surveillance data from Switzerland (inpatients and outpatients) show increasing numbers of CPE [27].

We found $27 \%$ of $P$. aeruginosa isolates to be carbapenem-resistant. This figure is comparable to data from 36 ICU in the United States which showed 35\% CRPA, mostly from the respiratory tract [28]. The association between institutional antibiotic consumption and detection of CRPA was not significant in the multivariable analysis. Indeed, previous studies have shown that total antibiotic consumption is less predictive of CRPA than the consumption of broad-spectrum antibiotics such as carbapenems [5]. In a recent study from selected ICU in Serbia, conducted between 2014 and 2018 , overall antibiotic consumption has been steadily decreasing, whereas the occurrence of CRAB and CRE increased [29]. On the other hand, in a before-after study from a French ICU, a reduction in quinolone and carbapenem use was associated with a decrease in CRPA, although total antibiotic use increased [30]. On the individual patient level, previous carbapenem exposure has also been identified as risk factor for CRPA [31].

A favourable trend was observed regarding MRSA in Swiss ICU. The fact that the decline in MRSA was exclusively attributable to haMRSA (and not to caMRSA) has been observed in other countries and has partly been attributed to increased awareness and infection control interventions, including the Dutch search-anddestroy approach [32]. Molecular typing is not routinely performed in Switzerland; this would allow to better discriminate haMRSA from caMRSA. In this context it is important to note that the concept of distinguishing haMRSA and caMRSA has been challenged in recent years, with traditional caMRSA such as USA30o increasingly detected in the healthcare setting and even showing a multi-resistant phenotype [33]. In contrast to MRSA, GRE have become more frequent, particularly in ICU from eastern Switzerland. This signal most probably reflects an interregional VRE outbreak caused by ST796 detected in 2017 [34].

Geographical differences have to be taken into account when explaining AMR trends in Switzerland. Historically, the south-western part of the country has been more affected by AMR than the east [10]. However, our data suggest that these differences are more and more levelling out. Not only were MRSA rates in 2018 very similar between the two regions, but CRE and also GRE were more common in the east than in the south-west. In addition, ICU in the east provided fewer bacterial isolates per represented bed than in the south-west (i.e. lower sampling density), which presumably led to further underestimation of resistance rates in the east. Antibiotic consumption was also somewhat lower in the south-west compared with the east for the year 2018. The reasons for this paradigm shift remain elusive. One (speculative) argumentation is that these favourable trends are a result of enhanced infection control measures implemented during the MRSA epidemic in south-western Switzerland.

Antibiotic consumption in Switzerland has traditionally been lower compared with other European countries [11]. Our data show that antibiotic consumption increased in Swiss ICU from 2009 to 2013. Similarly, data collected from German ICU between 2001 and 2015 have shown a steady increase in antibiotic consumption along with the above-mentioned increase in resistant Gram-negative bacteria [16]. However, consumption in Swiss ICU, including use of antibiotic substances on the 'watch' group of the WHO's AWaRe classification, declined between 2013 and 2018. Likewise, in countries of the European Union and the European Economic Area, overall use of cephalosporins, quinolones and 
carbapenems has been stabilising or even declining in the same time period [35].

Despite the decreased antibiotic use in intensive care, many antimicrobial stewardship activities are still lacking in a majority of Swiss hospitals [36]. Given the importance of rational antibiotic consumption in the control of AMR, the Swiss National Center for Infection Control launched an initiative to promote antimicrobial stewardship programmes (ASP) in Swiss hospitals in 2016 [37]. The aim was, among others things, to formulate requirements for institutional ASP and facilitate surveillance of antibiotic consumption and antibiotic resistance data using the ANRESIS database. In addition to ASP, particularly in light of the increasing numbers of resistant Gram-negative pathogens, we advocate for a multimodal infection control strategy to monitor and control the spread of AMR in ICU patients, including intensified admission screening programmes along with systematic molecular sequencing of pathogens, contact isolation of colonised or infected patients, strict adherence to standard precautions such as correct hand hygiene and use of personal protective equipment, and alertness to search and destroy environmental sources such as contaminated sink drainage systems, if necessary. We would like to emphasise that ASP (and also infection control measures) should not be reduced to human healthcare; a One Health approach should be pursued considering the complex interplay between human, animal and environmental health. Examples include the use of avoparcin as animal growth promoter in the 1990s, which may have contributed to the spread of glycopeptide-resistant genetic elements in humans [38], or the recent detection of carbapenemase-producing $E$. coli among employees of a Swiss veterinary clinic, which was probably due to cross-transmission from hospitalised companion animals [39].

An important limitation of this study was the lack of information on day of patient admission to the ICU. Although we excluded screening samples from the analysis, part of our data reflect antibiotic susceptibilities of samples collected at the time of ICU admission. This means that some resistant pathogens in our dataset were in fact community-acquired and were not a consequence of high institutional antibiotic consumption. We also suppose that restriction to bacterial samples collected $48 \mathrm{~h}$ after ICU admission would have resulted in even higher resistance rates. Furthermore, we could not distinguish between pathogens representing true infection and those only colonising. Also, because of the institution-specific patient identifier, we cannot exclude the possibility of duplicate isolates for patients being admitted to more than one ICU in the same year. Another limitation was the lack of information on individual antibiotic treatment before admission. This prevented us from including antibiotic consumption as a patient level variable in our hierarchical model.

\section{Conclusion}

Resistance among Enterobacterales in Swiss ICU is increasing. The rise in carbapenem resistance calls for a multimodal approach to improve surveillance (including systematic screening of ICU patients and molecular typing of resistant isolates) and adherence to standard infection control measures. Although antibiotic consumption is decreasing, the observed association between total antibiotic consumption and the occurrence of CRE underscores the need for antimicrobial stewardship in the Swiss intensive care setting.

\section{Acknowledgments}

We thank all ANRESIS laboratories and sentinel network participants for providing their data. Advisory board members of ANRESIS are: A. Cherkaoui, Bacteriology Laboratory, Geneva University Hospitals, Switzerland; O. Dubuis, Viollier AG, Basel, Switzerland; A. Egli, Clinical Microbiology, University Hospital Basel, Switzerland; V. Gaia, Department of microbiology, EOLAB, Bellinzona, Switzerland; S. Gottwalt, Federal Office of Public Health, Bern, Switzerland; A. Jayol, Etablissements Hospitaliers du Nord Vaudois, Yverdon, Switzerland; A. Kronenberg, Institute for Infectious Diseases, University of Bern, Switzerland; S.L. Leib, Institute for Infectious Diseases, University of Bern, Switzerland; J. Marschall, Department of Infectious diseases, University Hospital, Bern, Switzerland; H. Matter, Federal Office of Public Health, Bern, Switzerland; P. Nordmann, Molecular and Medical Microbiology, Department of Medicine, University Fribourg, Switzerland; V. Perreten, Institute of Veterinary Bacteriology, University of Bern, Switzerland; J.-C. Piffaretti, Interlifescience, Massagno, Switzerland; G. Prod'hom, Institute of Microbiology, Centre Hospitalier Universitaire Vaudois, Lausanne, Switzerland; K. Stärk, Federal Food Safety and Veterinary Office, Bern, Switzerland; J. Schrenzel, Bacteriology Laboratory, Geneva University Hospitals, Geneva, Switzerland; A. F. Widmer, Division of Infectious Diseases \& Hospital Epidemiology, University of Basel, Switzerland; G. Zanetti, Service of Hospital Preventive Medicine, Centre Hospitalier Universitaire Vaudois, Lausanne, Switzerland; R. Zbinden, Institute of Medical Microbiology, University of Zürich, Switzerland.

Funding: This work was supported by the research fund of the Cantonal Hospital St.Gallen. PK is funded by the Swiss National Science Foundation (grant No PZooP3_179919).

\section{Conflict of interest}

None declared.

\section{Authors' contributions}

P.K. conceived of the project. A.K. and M.S. helped supervise the project. S.B. developed the theory and performed the computations. F.B. verified the analytical methods. W.A., B.F., M.G., C.P., A.K. and M.S. contributed to the interpretation of the results. P.K. and S.B. wrote the manuscript with input from all authors.

\section{References}

1. Bassetti M, De Waele JJ, Eggimann P, Garnacho-Montero J, Kahlmeter G, Menichetti F, et al. Preventive and therapeutic strategies in critically ill patients with highly resistant bacteria. Intensive Care Med. 2015;41(5):776-95. https://doi. org/10.1007/s00134-015-3719-Z PMID: 25792203 
2. Vincent JL, Bihari DJ, Suter PM, Bruining HA, White J, Nicolas-Chanoin $\mathrm{MH}$, et al. The prevalence of nosocomial infection in intensive care units in Europe. Results of the European Prevalence of Infection in Intensive Care (EPIC) Study. JAMA. 1995;274(8):639-44. https://doi.org/10.1001/ jama.1995.03530080055041 PMID: 7637145

3. Holmes AH, Moore LSP, Sundsfjord A, Steinbakk M, Regmi S, Karkey A, et al. Understanding the mechanisms and drivers of antimicrobial resistance. Lancet. 2016;387(10014):17687. https://doi.org/10.1016/S0140-6736(15)00473-0 PMID: 26603922

4. Meyer E, Schwab F, Schroeren-Boersch B, Gastmeier P. Dramatic increase of third-generation cephalosporin-resistant E. coli in German intensive care units: secular trends in antibiotic drug use and bacterial resistance, 2001 to 2008. Crit Care. 2010;14(3):R113. https://doi.org/10.1186/cc9062 PMID: 20546564

5. Plüss-Suard C, Pannatier A, Kronenberg A, Mühlemann K, Zanetti G. Impact of antibiotic use on carbapenem resistance in Pseudomonas aeruginosa: is there a role for antibiotic diversity? Antimicrob Agents Chemother. 2013;57(4):1709-13. https://doi.org/10.1128/AAC.01348-12 PMID: 23357763

6. Tacconelli E, Carrara E, Savoldi A, Harbarth S, Mendelson M, Monnet DL, et al. Discovery, research, and development of new antibiotics: the WHO priority list of antibiotic-resistant bacteria and tuberculosis. Lancet Infect Dis. 2018;18(3):318-27. https:// doi.org/10.1016/S1473-3099(17)30753-3 PMID: 29276051

7. World Health Organization (WHO). Antibiotic resistance. Key facts. Geneva: WHO: 2020. Available from: https://www.who. int/news-room/fact-sheets/detail/antibiotic-resistance

8. Lemmenmeier E, Kohler P, Bruderer T, Goldenberger D, Kleger G-R, Schlegel M. First documented outbreak of KPC-2producing Klebsiella pneumoniae in Switzerland: infection control measures and clinical management. Infection. 2014;42(3):529-34. https://doi.org/10.1007/s15010-013-05789 PMID: 24477886

9. Kohler P, Fulchini R, Albrich WC, Egli A, Balmelli C, Harbarth S, et al. Antibiotic resistance in Swiss nursing homes: analysis of National Surveillance Data over an 11-year period between 2007 and 2017. Antimicrob Resist Infect Control. 2018;7(1):88 https://doi.org/10.1186/s13756-018-0378-1 PMID: 30038781

10. Olearo F, Albrich WC, Vernaz N, Harbarth S, Kronenberg A, Swiss C, Swiss Centre For Antibiotic Resistance Anresis. Staphylococcus aureus and methicillin resistance in Switzerland: regional differences and trends from 2004 to 2014. Swiss Med Wkly. 2016;146:W14339. https://doi. org/10.4414/smw.2016.14339 PMID: 27631162

11. Plüss-Suard C, Pannatier A, Kronenberg A, Mühlemann K, Zanetti G. Hospital antibiotic consumption in Switzerland: comparison of a multicultural country with Europe. J Hosp Infect. 2011;79(2):166-71. https://doi.org/10.1016/j. jhin.2011.05.028 PMID: 21820207

12. Clinical and Laboratory Standards Institute (CLSI). Global Laboratory Standards for a Healthier World. Wayne: CLSI. [Accessed: 8 Nov 2021]. Available from: http://www.clsi.org

13. European Committee on Antimicrobial Susceptibility Testing (EUCAST). Breakpoint tables for interpretation of MICS and zone diameters. Version 11.0, valid from 2021-01-01. Växjö: EUCAST; 2021. Available from: https://www.eucast. org/fileadmin/src/media/PDFs/EUCAST_files/Breakpoint_ tables/v_11.0_Breakpoint_Tables.pdf

14. World Health Organization (WHO) Collaborating Centre for Drug Statistics Methodology. DDD alterations from 20052020. Oslo: Norwegian Institute of Public Health. [Accessed: 7 Jul 2020]. Available from: https://www.whocc.no/ atc ddd alterations cumulative/ddd alterations

15. World Health Organization (WHO). AWaRe - List of antibiotics. Geneva: WHO. [Accessed: 6 May 2020]. Available from: https:// aware.essentialmeds.org/list

16. Remschmidt C, Schneider S, Meyer E, Schroeren-Boersch B, Gastmeier P, Schwab F. Surveillance of antibiotic use and resistance in intensive care units (SARI). Dtsch Arztebl Int. 2017;114(50):858-65. https://doi.org/10.3238/ arztebl.2017.0858 PMID: 29271345

17. Arnaud I, Maugat S, Jarlier V, Astagneau P, National Early Warning, Investigation and Surveillance of HealthcareAssociated Infections Network (RAISIN)/multidrug resistance study group. Ongoing increasing temporal and geographica trends of the incidence of extended-spectrum beta-lactamaseproducing Enterobacteriaceae infections in France, 2009 to 2013 . Euro Surveill. 2015;20(36):30014. https://doi. org/10.2807/1560-7917.ES.2015.20.36.30014 PMID: 26536042

18. Iacchini S, Sabbatucci M, Gagliotti C, Rossolini GM, Moro $\mathrm{ML}$, Iannazzo S, et al. Bloodstream infections due to carbapenemase-producing Enterobacteriaceae in Italy: results from nationwide surveillance, 2014 to 2017. Euro Surveill.
2019;24(5):1800159. https://doi.org/10.2807/1560-7917. ES.2019.24.5.1800159 PMID: 30722813

19. Kronenberg A, Hilty M, Endimiani A, Mühlemann K. Temporal trends of extended-spectrum cephalosporin-resistant Escherichia coli and Klebsiella pneumoniae isolates in inand outpatients in Switzerland, 2004 to 2011. Euro Surveill. 2013;18(21):20484. https://doi.org/10.2807/ese.18.21.20484en PMID: 23725981

20. Federal Office of Public Health and Federal Food Safety and Veterinary Office. Swiss antibiotic resistance report 2018. Usage of Antibiotics and occurrence of antibiotic resistance in bacteria from humans and animals in Switzerland. FOPH publication number: 2018-OEG-87; 2018. Available from: https://www.bag.admin.ch/dam/bag/de/dokumente/mt/star/ swiss-antibiotic-resistance-report-2018.pdf

21. Falagas ME, Tansarli GS, Karageorgopoulos DE, Vardakas KZ. Deaths attributable to carbapenemresistant Enterobacteriaceae infections. Emerg Infect Dis. 2014;20(7):1170-5. https://doi.org/10.3201/eid2007.121004 PMID: 24959688

22. Babouee Flury B, Ellington MJ, Hopkins KL, Turton JF, Doumith $M$, Loy R, et al. Association of novel nonsynonymous single nucleotide polymorphisms in ampD with cephalosporin resistance and phylogenetic variations in ampC, ampR, ompF, and ompC in Enterobacter cloacae isolates that are highly resistant to carbapenems. Antimicrob Agents Chemother. 2016;60(4):2383-90. https://doi.org/10.1128/AAC.02835-15 PMID: 26856839

23. Gomez-Simmonds A, Annavajhala MK, Wang Z, Macesic N, Hu Y, Giddins MJ, et al. Genomic and geographic context for the evolution of high-risk carbapenem-resistant Enterobacter cloacae complex clones ST171 and ST78. MBio. 2018;9(3):e00542-18. https://doi.org/10.1128/mBio.00542-18 PMID: 29844109

24. Seiffert SN, Wüthrich D, Gerth Y, Egli A, Kohler P, Nolte O. First clinical case of KPC-3-producing Klebsiella michiganensis in Europe. New Microbes New Infect. 2019;29:100516. https:// doi.org/10.1016/j.nmni.2019.100516 PMID: 30949345

25. David S, Reuter S, Harris SR, Glasner C, Feltwell T, Argimon S, et al. Epidemic of carbapenem-resistant Klebsiella pneumoniae in Europe is driven by nosocomial spread. Nat Microbiol. 2019;4(11):1919-29. https://doi.org/10.1038/s41564-019-04928 PMID: 31358985

26. Kizny Gordon AE, Mathers AJ, Cheong EYL, Gottlieb T, Kotay S, Walker AS, et al. The hospital water environment as a reservoir for carbapenem-resistant organisms causing hospital-acquired infections-a systematic review of the literature. Clin Infect Dis. 2017;64(10):1435-44. https://doi.org/10.1093/cid/cix132 PMID: 28200000

27. Ramette A, Gasser M, Nordmann P, Zbinden R, Schrenzel $J$, Perisa D, et al. Temporal and regional incidence of carbapenemase-producing Enterobacterales, Switzerland, 2013 to 2018. Euro Surveill. 2021;26(15):1900760. https:// doi.org/10.2807/1560-7917.ES.2021.26.15.1900760 PMID: 33860749

28. Asempa TE, Nicolau DP, Kuti IL. Carbapenem-nonsusceptible Pseudomonas aeruginosa isolates from intensive care units in the United States: a potential role for new $\beta$-Lactam combination agents. J Clin Microbiol. 2019;57(8):e00535-19. https://doi.org/10.1128/JCM.00535-19 PMID: 31118271

29. Popović R, Tomić Z, Tomas A, Anđelić N, Vicković S, Jovanović $\mathrm{G}$, et al. Five-year surveillance and correlation of antibiotic consumption and resistance of Gram-negative bacteria at an intensive care unit in Serbia. J Chemother. 2020;32(6):294303. https://doi.org/10.1080/1120009X.2020.1755588 PMID: 32321359

30. Abbara S, Pitsch A, Jochmans S, Hodjat K, Cherrier $\mathrm{P}$, Monchi $\mathrm{M}$, et al. Impact of a multimodal strategy combining a new standard of care and restriction of carbapenems, fluoroquinolones and cephalosporins on antibiotic consumption and resistance of Pseudomonas aeruginosa in a French intensive care unit. Int J Antimicrob Agents. 2019;53(4):416-22. https://doi.org/10.1016/j. ijantimicag.2018.12.001 PMID: 30537533

31. Labaste F, Grossac J, Bounes FV, Conil J-M, Ruiz S, Seguin T, et al. Risk factors for acquisition of carbapenem-resistance during treatment with carbapenem in the intensive care unit: a prospective study. Eur I Clin Microbiol Infect Dis. 2019;38(11):2077-85. https://doi.org/10.1007/s10096-01903644-6 PMID: 31482416

32. Khatib R, Sharma M, Iyer S, Fakih MG, Obeid KM, Venugopal $A$, et al. Decreasing incidence of Staphylococcus aureus bacteremia over 9 years: greatest decline in communityassociated methicillin-susceptible and hospital-acquired methicillin-resistant isolates. Am J Infect Control. 2013;41(3):210-3. https://doi.org/10.1016/j.ajic.2012.03.038 PMID: 23040608 
33. Klein S, Hannesen J, Zanger P, Heeg K, Boutin S, Nurjadi D. Entry of Panton-Valentine leukocidin-positive methicillinresistant Staphylococcus aureus into the hospital: prevalence and population structure in Heidelberg, Germany 2015-2018. Sci Rep. 2020;10(1):13243. https://doi.org/10.1038/s41598020-70112-Z PMID: 32764618

34. Wassilew N, Seth-Smith HM, Rolli E, Fietze Y, Casanova C, Führer U, et al. Outbreak of vancomycin-resistant Enterococcus faecium clone ST796, Switzerland, December 2017 to April 2018. Euro Surveill. 2018;23(29):1800351. https://doi. org/10.2807/1560-7917.ES.2018.23.29.1800351 PMID: 30043725

35. Peñalva G, Högberg LD, Weist K, Vlahović-Palčevski V, Heuer 0 , Monnet DL, et al. Decreasing and stabilising trends of antimicrobial consumption and resistance in Escherichia coli and Klebsiella pneumoniae in segmented regression analysis, European Union/European Economic Area, 2001 to 2018. Euro Surveill. 2019;24(46):1900656. https://doi.org/10.2807/15607917.ES.2019.24.46.1900656 PMID: 31771708

36. Osthoff M, Bielicki J, Widmer AF-X, For Swissnoso. Evaluation of existing and desired antimicrobial stewardship activities and strategies in Swiss hospitals. Swiss Med Wkly. 2017;147:W14512. PMID: 29063523

37. Swissnoso. Teilprojekt Antibiotika Stewardship Programme. [Subproject antibiotic stewardship programme]. Bern: Swissnoso. [Accessed: 3 Jul 2021]. German. Available from: https://www.swissnoso.ch forschung-entwicklung/umsetzung-star/teilprojekte/ teilprojekt-antibiotika-stewardship-programme

38. Acar J, Casewell M, Freeman J, Friis C, Goossens H. Avoparcin and virginiamycin as animal growth promoters: a plea for science in decision-making. Clin Microbiol Infect. 2000;6(9):477-82. https://doi.org/10.1046/j.14690691.2000.00128.x PMID: 11168181

39. Endimiani A, Brilhante M, Bernasconi OJ, Perreten V, Schmidt JS, Dazio V, et al. Employees of Swiss veterinary clinics colonized with epidemic clones of carbapenemase-producing Escherichia coli. J Antimicrob Chemother. 2020;75(3):766-8. https://doi.org/10.1093/jac/dkz470 PMID: 31819979

\section{License, supplementary material and copyright}

This is an open-access article distributed under the terms of the Creative Commons Attribution (CC BY 4.0) Licence. You may share and adapt the material, but must give appropriate credit to the source, provide a link to the licence and indicate if changes were made.

Any supplementary material referenced in the article can be found in the online version.

This article is copyright of the authors or their affiliated institutions, 2021. 